\title{
The Need of Sealants in Oral Health
}

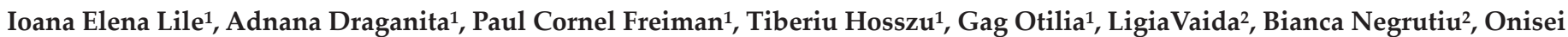 \\ Doina $^{1 *}$
}

${ }^{1}$ Western University "Vasile Goldis" of Arad, Faculty of Medicine, Pharmacy and Dental Medicine

${ }^{2}$ University of Oradea, Faculty of Medicine and Pharmacy, Dental Medicine II

Correspondence to: Onisei Doina, Western University "Vasile Goldis" of Arad, Faculty of Medicine, Pharmacy and Dental Medicine, Romania;

Email: doina_onisei@yahoo.com

Received: June 08, 2018; Accepted: June 18, 2018; Published: June 20, 2018;

\begin{abstract}
A good oral health education program starts from early ages, and the most important time to initiate this it is when the first permanent tooth (the first permanent molar) erupts, and it lies on a correct diet and hygiene habits, including a healthy diet, a correct brushing technique, and a preventive therapeutic approach. The beneficial effect of a preventive program is a good oral health and the therapeutic approach is the dental sealing. Of course it is important to motivate small patients to maintain a good oral health, through the correct use of oral hygiene products, individual brushing techniques, and good eating habits. The prevention programs and the children's receptiveness in maintaining proper oral hygiene can improve the oral health. If there is good communication between the physician and the patient then we can talk about the triad: theoretical presentation, practical exercises and individual biofilm removal techniques, which are indispensable factors in achieving an appropriate oral health status. When dental education programs can't provide the proper prophylaxis of caries lesion, especially at the occlusal surfaces of molars and premolars with deep and retentive pits and grooves, dental sealing is an ideal option.
\end{abstract}

Keywords: Dental Plaque, Dental Sealing, Tooth Decay, Plaque Indexes, Biofilm

\section{Introduction}

Risk assessment for the disease development is an essential component of any oral disease prevention program. Susceptibility risks can be determined by various methods including the person, the whole community and of course the teeth, and all dental surfaces. Tooth decay is a bacterial-dependent disease so dental sealing is a preventive measure that can be implemented when the patient is at risk. There are many risk factors that can influence the formation of carious lesions which includes the eating habits and dental hygiene habits, regarding brushing techniques in sense of frequency and time of dental brushing. Of course that periodic consultation and early detection of caries are also important factors that hold the key of success in dental health. Oral disorders, starting from the incipient lesions and leading to the most complicated clinical cases, depend on many factors including the dietetic and hygiene habits of the patient. When talking about dietetic habits, the evolution of the carious lesion depends to a great extent, of the presence in the diet of sugars, carbohydrates (juices) etc., but also it depends on regular meals without eating between meals. Also, an important factor in the appearance and evolution of the carious lesion is the patient's hygiene habits, such as how he performs dental brushing, how many times a day, etc. In other words good hygiene habits must be learned from an early age, and this can be done through dental education programs, which can be implemented in schools. Tooth brushing is an essential part of an effective dental education program, and achieving optimal oral health depends on the efficiency of the method used to remove the biofilm from the surface of the teeth. Tooth brushing aims, to remove, by mechanical means, the food residues and the biofilm from dental surfaces. The efficiency of the biofilm removal depends on several factors: the time of dental brushing, its frequency, the technique used, the tools used, and the quality of the toothpastes. Associating oral hygiene with a healthy diet will help reduce the risk of carious lesions.

Even if all of these rules are respected by patients, this may not be enough, if the teeth have deep and retentive pits and fissures, because the toothbrush bristle can't get inside those grooves, and clean the biofilm, which can accumulate and determine the lesion on the occlusal surface of the premolars and molars. Dental sealing is considered by the World Health Organization to be a major factor in the prevention of carious lesions, especially in schools with children from low-income families, so through dental sealing programs it can be reduce the incidence of caries, especially when focusing on sealing permanent molars in children. Preventive dental sealing treatment has a beneficial effect on the patient, from all points of view. Maintaining a good oro-dental health is one of the most important therapeutic attitudes in all dentistry branches. The most beautiful feeling as a dentist is when he succeeds in motivating the patient about oral health, because it is easier to prevent than to treat a disease.

\section{Methods \& Materials}

In our study we conducted a preventive school program, which included theoretical presentation, practical exercises, individual biofilm removal techniques and dental sealing. We randomly selected a group of 107 children who received specialized consultations. So, the study was conducted on a group of 107 patients, with age range 
between 6-8 years, from an urban school, class 0 and 1 students. We evaluated the ways in which living habits, influence the oral health, so we gathered data about hygiene habits and eating habits. In this part of the study, we evaluated the foods that the patient usually consumes and which may have repercussions on oral health, but also if the patient consumes sugars, sweet snacks, carbohydrate beverages, sweets (chocolate), fruits, fats and vegetables, and the way they do it. (Figure 1)

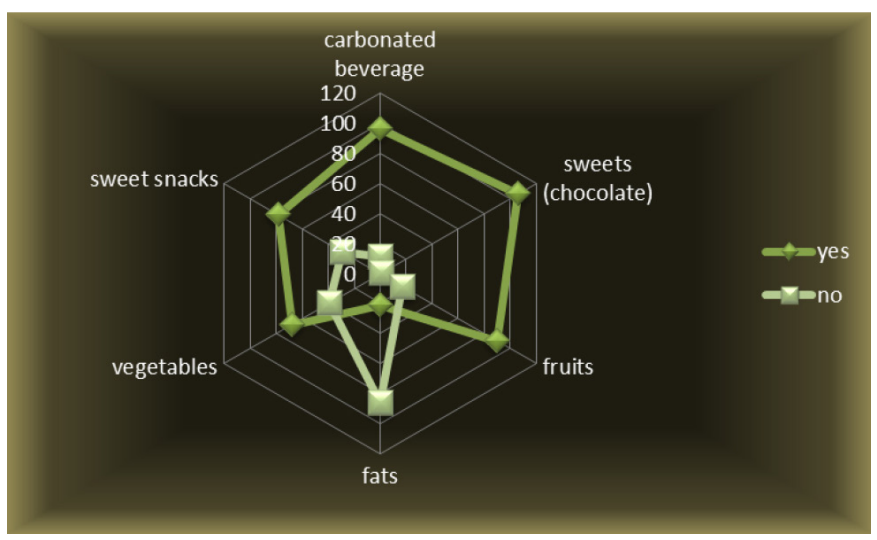

Figure1. Eating habits.

It is noted that from the entire group of patients, the majority of children consume carbonated beverages, which is not suited for a good oral health status. However, a very small percentage of children do not consume carbonated beverages. Drinking this kind of beverage, is not a good thing for oral health, especially if dental brushing is not done right after, so we have gathered data on sweets consumption, and we noticed that all children in the batch eat sweets except for one boy, which is worrying in the absence of dental brushing. It's a concerning result for children's oral hygiene, but if teeth brushing is performed after eating sweets, then the oral health of small children will not be affected. We next correlated the data on fruit consumption, which is a part of the category of foods that are beneficial to both the oro-dental health and the health of the whole organism. It is observed that most of the patients included in the lot consume fruits, almost in the same way that they consume carbonated beverage (Figure1). The data collected about fat consumption, which, at some point is beneficial to dental health, but can also have a negative effect to oral health, is as follow: an increased percentage of patients consume non-fat foods, and a small number of patient have fat in the diet. The consumption of vegetables, which are recognized as being good for oral health, is found in more than half of the patients; in fact a third does not consume them against the two-thirds who consume them. This result is a positive one, because there are more children who consume vegetables, but it would have been better if that number would have been even higher.

After correlating all the outcomes on the patients' diet, we assessed the way in which the small children consume sugars, so we evaluated whether eating sugary foods is done between or during meals (Figure 2).

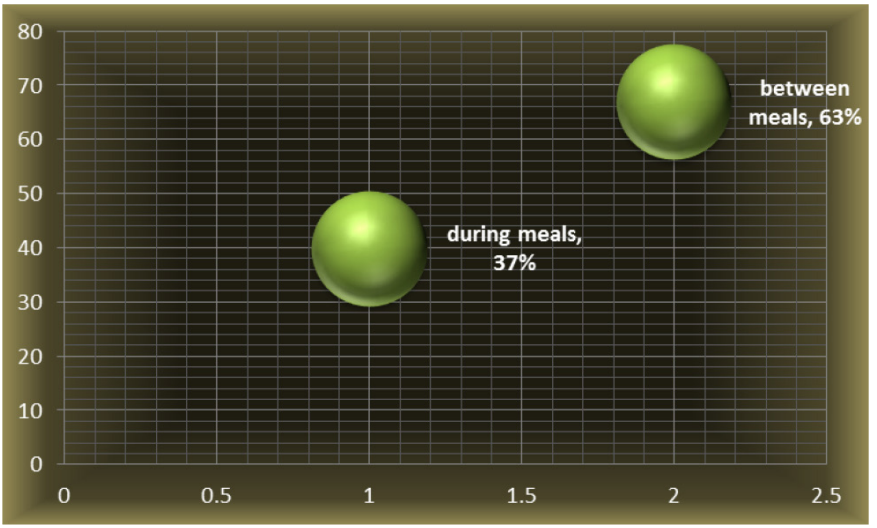

Figure 2. Eating habits-consumed sugars.

Sugar is consumed between meals by most patients in the group, regardless of gender, representing a high percentage, $63 \%$, with a total of 67 patients, compared with 40 patients who consume sugar during meals, representing $37 \%$ of the children. This result is not satisfactory because the consumption of sugary foods increases the risk of carious lesions in absence of dental brushing after consumption. We have noticed that patients or their parents give a great importance to oral hygiene products by choosing different products on the market but they do not put as much emphasis on the brush technique and its frequency. It is noted that all patients include in their hygiene habits, the toothbrush and toothpaste to perform everyday dental brushing. Most of the patients tend to complete their daily dental hygiene with the mouth wash, which chemically removes the biofilm from dental surfaces, so there was a $62 \%$ of children, male and female that uses mouthwash, this being a relatively satisfactory percentage, but it would have been better if this percentage were higher. Regarding dental floss, things are different, and it appears that $85 \%$ of patients do not use it. This result is not satisfactory one because the use of dental floss reduces the risk of carious lesions on the proximal surfaces of the teeth (Figure 3).

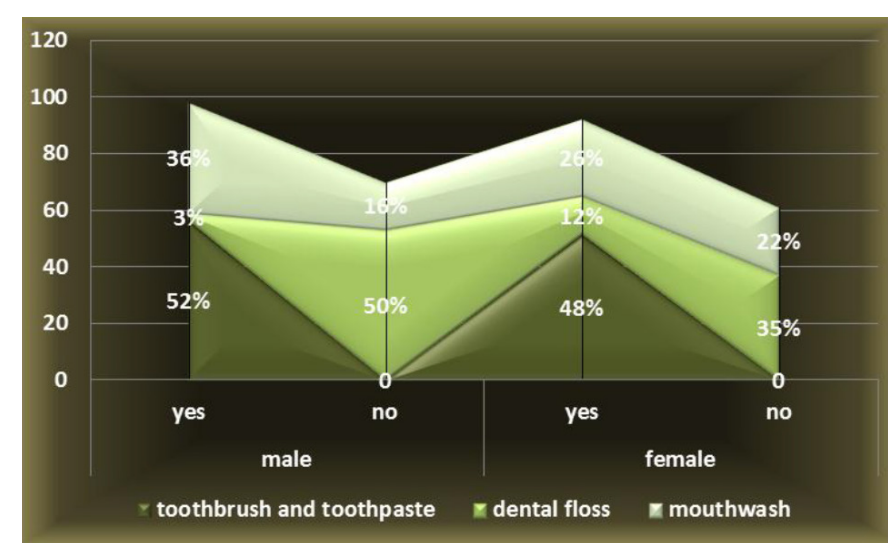

Figure 3. Products used in dental hygiene habits.

Although all the patients included in the study use the toothpaste and the toothbrush, a special importance should be given to the frequency of dental brushing. It is noted that most patients perform 
dental brushing twice a day, accounting for 57 patients of both sexes, namely $28 \%$ of male patients and $25 \%$ of female patients, followed by $29 \%$ of patients performing dental care after each meal, $13 \%$ patients performing dental brushing once a day, and the smallest percentage is $5 \%$ representing patients who do not perform dental brushing every day (Figure 4).

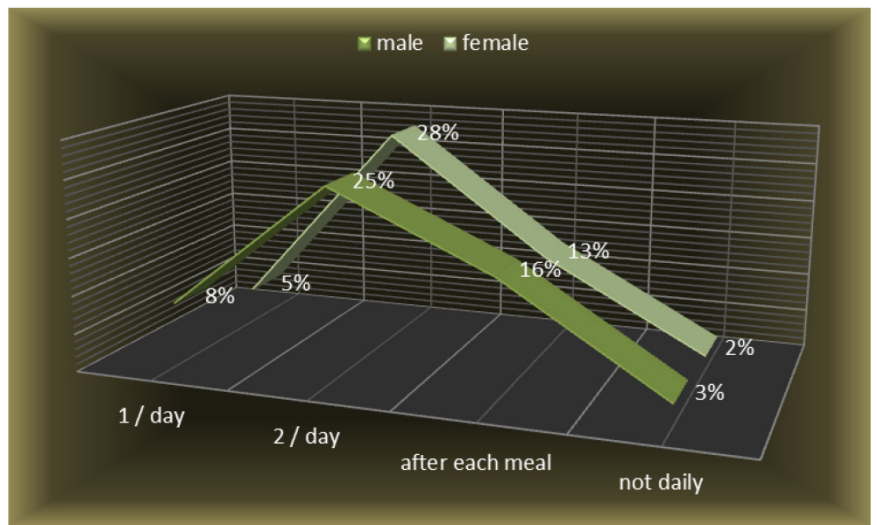

Figure 4. The frequency of dental brushing.

Following the correlation of all the results obtained so far, the tendency of the entire group of patients relating to oral hygiene habits and diet habits are in ascending parameters, towards good oral health, but some improvements should be made. The gaps that still exist in the habits of the patients had repercussions on oral health, so initial patient evaluation did not necessarily have good results. In the initial examination of the patients, they presented different degrees of oral hygiene, as can be seen in the chart below, $11 \%$ boys and $7 \%$ girls were with unsatisfactory hygiene, $25 \%$ boys and $22 \%$ girls, had an average grade of hygiene and good hygiene was achieved by $16 \%$ boys and $19 \%$ of the girls. According to the chart below (Figure 5), the majority of patients, $47 \%$, had average oral hygiene, followed by the $35 \%$ with good hygiene, and the lowest percentage was for unsatisfactory hygiene, $18 \%$.

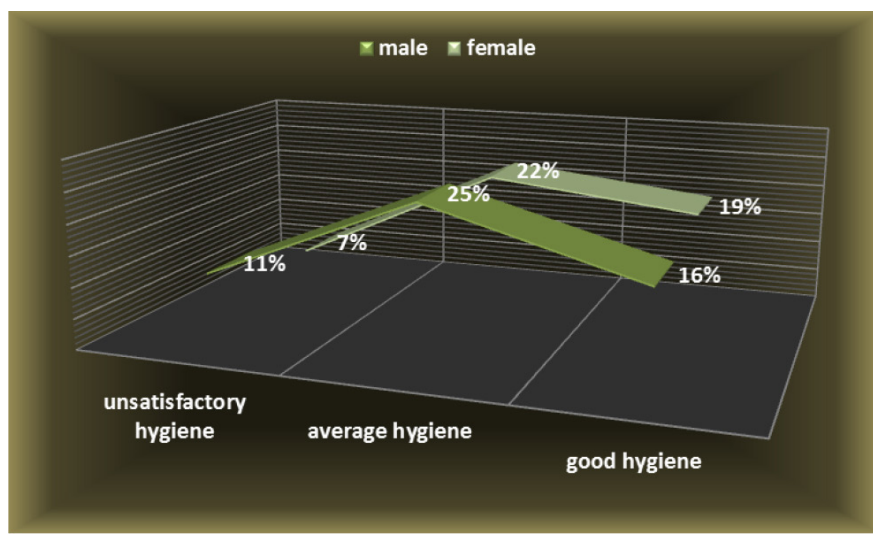

Figure 5. The degree of oral hygiene.

The idea deduced from this series of arguments or findings is that the percentage of patients with unsatisfactory grade of hygiene was $18 \%$ patients, and $82 \%$ of children had average or good hygiene (Figure5). By correlating all data relating to dietary habits, dental brushing with its frequency, the auxiliary means used in removing dental plaque and the evaluation of oral hygiene, we tried to relate them to the incidence of caries in permanent teeth of this children, namely the first molars.

From the data provided in the graph below (Figure 6), it is found that a number of 226 teeth are without lesions or with clinically undetected lesions and it represents $53 \%$ of teeth, more than half of the teeth examined. Also we found that $19 \%$ of the molars had incipient lesions. Tooth decay was present in an $28 \%$ of the molars examined, but $9 \%$ of molars were treated before our examination, and $19 \%$ still presented caries lesions. In other word we can say that tooth decay was present in $15 \%$ of males and $13 \%$ females.

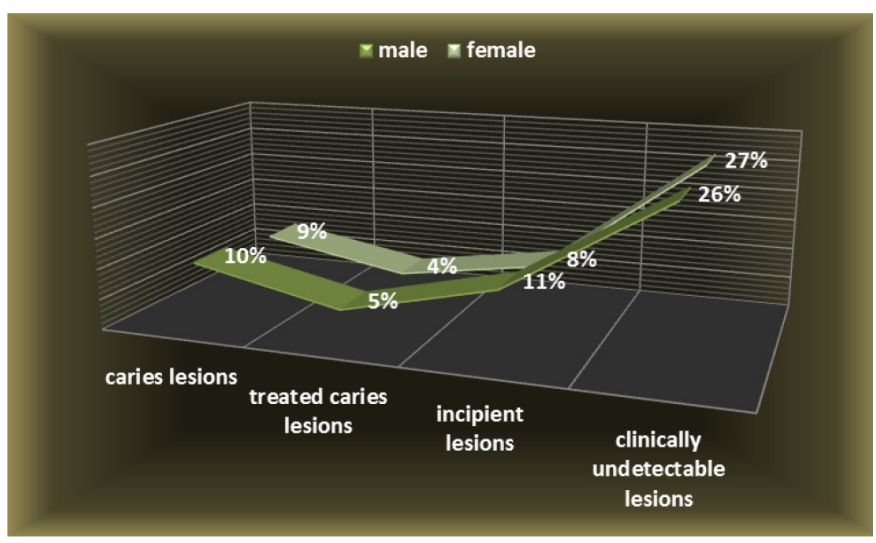

Figure 6. Incidence of caries lesions at first molars.

From the category of teeth with no clinically detectable lesions, 121 of them had a risk of carious lesions, representing $28 \%$ of the whole teeth we examined, and it is shown in the chart below (Figure 7).This percentage does not differ significantly from that of teeth without risk to caries, which is $25 \%$ of examined teeth.

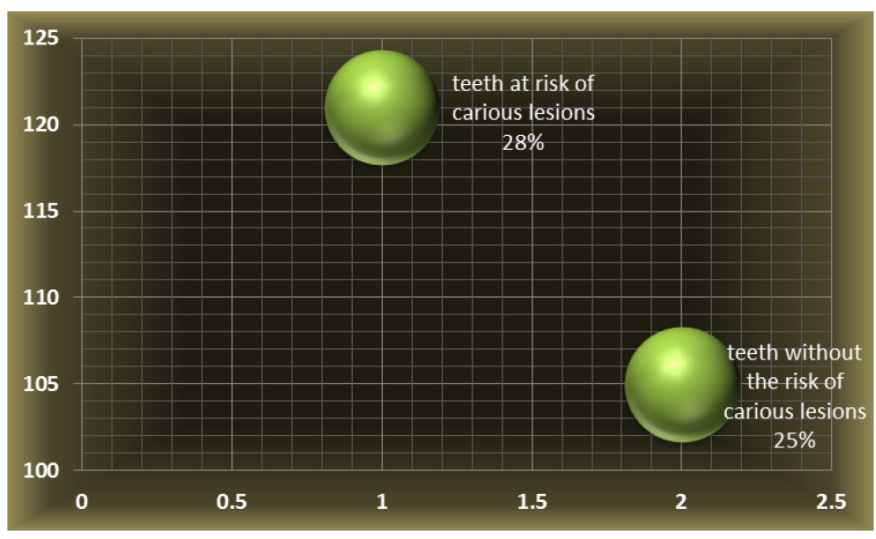

Figure7. Teeth at risk of carious lesions.

The fact that there is not a very big difference between these percentages suggests that there is a high risk of tooth decay among children with age range between 6-8 year old. Following the results of the incidence of carious lesions and the risk of their occurrence, all the teeth presenting a risk of carious lesions have been sealed. Those teeth also presented deep retentive pits and fissures on the occlusal surfaces or incipient lesions, undetectable on X-ray, in the form of demineralized enamel, shown as white spots. Teeth sealed who 
presented deep retentive pits and fissures were 89 and there were 32 teeth sealed with white spots, incipient lesions, undetectable on X-ray, in the form of demineralized enamel. From the whole number of teeth that had dental sealing there were $74 \%$ with deep and retentive pits and fissures, and $26 \%$ of teeth sealed were with white spots. (Figure 8 )

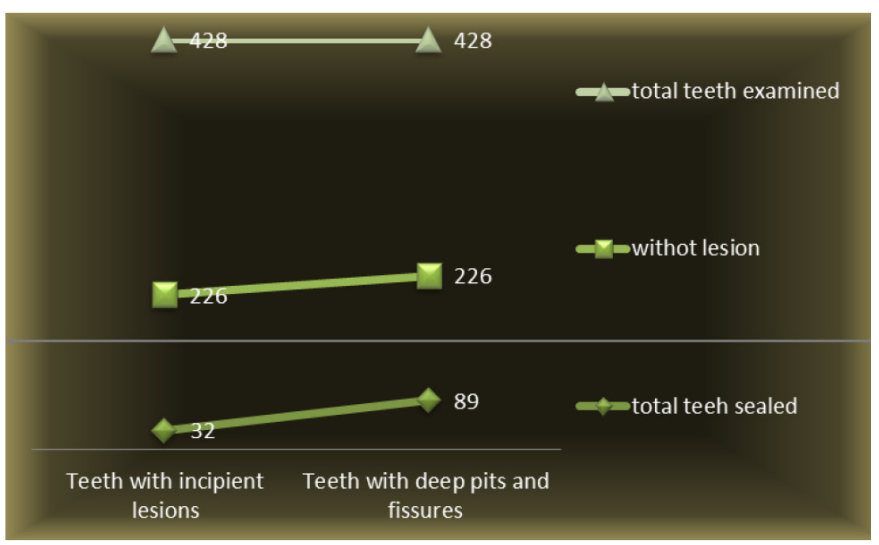

Figure 8. Teeth examined and sealed.

From the graph above, it is noted that the total number of 428 teeth examined, only 226 did not show treated or untreated dental lesions, so only this half was evaluated for dental sealing. The other half who experienced lesions with or without treatment was examined and either the incorrect dental restorations were replaced or the present lesions were treated. From the evaluated teeth for dental sealing, which is, half of the teeth evaluated and examined in the entire group of patients, the need for dental sealing was at half of them. This means that of the 226 teeth evaluated in terms of prophylactic and preventive treatment, 121 teeth, permanent first molars, had an indication of sealing.

The teeth which received dental sealing were half of the total teeth not affected by lesions, and this is a very high number, because in fact, these teeth actually have a tooth decay predisposition, and in the absence of dental sealing they lose their morpho structural integrity. Following control, 6 months after application, most of the seals remained unchanged, however 3 dental seals were no longer present on the teeth, and in the control after 1 year, 5 teeth lost their sealing. The loss of dental sealing may vary, depending on whether the patients followed the indication for a good oral health or simply because of local factors. It is noted that the number of un affected dental sealing is high, so the purpose of prevention is met. There was also an improvement in oral health through better diet habits and oral hygiene habits with better brushing techniques and increasing brushing frequency, and also using more auxiliary means for removing the biofilm.

\section{Conclusion}

Dental sealing has had a good result because as long as it remained in the pits and fissures, its preventive purpose was fulfilled. The need for dental sealing, as shown in our study is quite high, and depends on many factors, such as better brushing techniques, increasing brushing frequency, using more auxiliary means such as dental floss and mouthwash, in removing the biofilm, through both local as well as general means.

\section{References}

1. American Academy of Pediatric Dentistry (2012) Guideline on caries-risk assessment and management for infants, children, and adolescents. Pediatr Dent 34: $118-125$.

2. Amin HE1 (2008) Clinical and antibacterial effectiveness of three different sealant materials. J Dent Hyg 82: 45. [crossref]

3. Dye BA, Mitnik GL, Iafolla TJ, Vargas CM (2017) Trends in dental caries in children and adolescents according to poverty status in the United States from 1999 through 2004 and from 2011 through 2014. J Am Dent Assoc 148: 550-565. [crossref]

4. Dye BA, Thornton-Evans G, Li X, Iafolla TJ (2015) Dental caries and sealant prevalence in children and adolescents in the United States, 2011-2012. NCHS Data Brief 191: 1-8. [crossref]

5. Griffin SO1, Gray SK, Malvitz DM, Gooch BF (2009) Caries risk in formerly sealed teeth. J Am Dent Assoc 140: 415-423. [crossref]

6. Lile IE, Freiman PC, Hosszu T, Vasca E, Vasca V, et al. (2015) A Subsidiary Physical Research of Glass Ionomers. Jurnal Medical Aradean 52: 175-179.

7. Tagliaferro EP, Pardi V, Ambrosano GM, Meneghim Mde C, da Silva SR, et al. (2011) Occlusal caries prevention in high and low risk schoolchildren. A clinical trial. Am J Dent 24: 109-114. [crossref]

8. Tellez M, Gray SL, Gray S, Lim S, Ismail AI (2011) Sealants and dental caries: dentists' perspectives on evidence-based recommendations. JADA 142: 1033-1040.

9. Vaida L, Moldovan L, Lile IE, Todor BI, Porumb A, et al. (2015) A Comparative Study on Mechanical Properties of Some Thermoplastic and Thermo Set Resins Used for Orthodontic Appliances, MaterialePlastice 52: 364-367

10. Wright JT, Tampi MP, Graham L (2016) Sealants for preventing and arresting pitand-fissure occlusal caries in primary and permanent molars: a systematic review of randomized controlled trials a report of the American Dental Association and the American Academy of Pediatric Dentistry. JADA 147: 631-645.

\section{Citation:}

Ioana Elena Lile, Adnana Draganita, Paul Cornel Freiman, Tiberiu Hosszu, Gag Otilia, LigiaVaida, Bianca Negrutiu, Onisei Doina (2018) The Need of Sealants in Oral Health. J Dent Maxillofacial Res Volume 1(1): 1-4. DOI: $10.31038 / J D M R .1000101$ 